\title{
Revisiting phase transitions in Ca-modified lead titanate ceramics using synchrotron XRD
}

\author{
Flávia R. Estrada1, Larissa Galão ${ }^{1,2}$, Ducinei Garcia ${ }^{3}$ \\ ${ }^{1}$ Brazilian Synchrotron Light Laboratory, Giuseppe Máximo Scolfaro, 10000, Campinas - SP, Brazil, \\ 2 “Gleb Wataghin” Institute of Physics, State University of Campinas, R. Sérgio Buarque de Holanda, 777, Campinas - SP, Brazil, \\ ${ }^{3}$ Physics Department, Federal University of São Carlos, São Carlos - SP, Brazil.
}

\section{flavia.estrada@lnls.br}

Lead titanate $\left(\mathrm{PbTiO}_{3}\right)$ is a prototype ferroelectric material. At high temperatures, it presents the ideal centrosymmetric cubic perovskite structure with $P m-3 m$ symmetry. Cooling down to $770 \mathrm{~K}$, a first-order phase transition from the paraelectric phase to the non-centrosymmetric tetragonal $P 4 \mathrm{~mm}$ is observed [1]. Its high para-ferroelectric phase transition temperature would be promising for its technological application. However, the high anisotropy $(6 \% \mathrm{c} / \mathrm{a}$ at room temperature) combined with its positive thermal expansion in cooling, makes it impossible to produce this ceramic in the form of bulk. Doping the $\mathrm{Pb}$ or Ti site of the perovskite are options to decrease the anisotropy only enough to makes possible bulk production [2]. In this work, we study the effect of isovalent substitution of $\mathrm{Pb}^{+2}$ by $\mathrm{Ca}^{+2}$ in paraelectric to ferroelectric phase transition by structural, dielectric, and ferroelectric properties.

The bulk ceramics with stoichiometry $\mathrm{Pb}_{0.6} \mathrm{Ca}_{0.4} \mathrm{TiO}_{3}$ were synthesized by solid-state reaction, uniaxially and hydrostatically pressured and sintered by the conventional method. The electric permittivity as a function of frequency and temperature was carried out in an impedance analyzer (Agilent - 4294A) from $20 \mathrm{~K}$ up to $600 \mathrm{~K}$ with the sample in Linkan furnace and APD cryostat. The ferroelectric polarization versus electric field loops were carried out using a Sawyer-Tower circuit and APD cryostat. To perform the Synchrotron X-ray diffraction (SXRD) patterns collection a piece of the bulk was crushed into thin powder and annealed at $600 \mathrm{~K}$ for 5 hours to remove the residual strain. The patterns were collected at XPD and XRD1 beamlines at the Brazilian Synchrotron Laboratory. The high-temperature piece of experiment was performed in reflection geometry using Arara's furnace, while the low-temperature piece of experiment was performed in transmission mode using Oxford Cryoject.

The structural analyses were performed by Rietveld Refinement method using GSASII and the model $\mathrm{P} 4 \mathrm{~mm}$ from the pristine $\mathrm{PbTiO}_{3}$ compound. The lattice parameters as a function of temperature are presented in Figure 1. Heating up, the linear expansion of the $a$ lattice parameter is observed while $c$ contracts until $360 \mathrm{~K}$, then an abrupt change happens and $c$ presents a linear expansion. This structural anomaly temperature is relatively close to the paraelectric-ferroelectric phase transition is observed at $\sim 390 \mathrm{~K}$ by ferroelectric and dielectric properties [3]. However, differently to the pristine $\mathrm{PbTiO}_{3}$ compound, this phase transition happens from a tetragonal noncentrosymmetric to another tetragonal but centrosymmetric (therefore paraelectric) symmetry which can be the same phase of $\mathrm{CaTiO}_{3}$-high temperature $(\mathrm{I} / \mathrm{mcm})$ [4].

Moreover, uniaxial anisotropy was observed in the ferroelectric phase, in the direction (001), as represented by the peak width difference between (002) and (200) peaks inset of figure 1. This anisotropy became isometric at the paraelectric phase.

To conclude, from $40 \%$ of Ca-doping in $\mathrm{PbTiO}_{3}$ ceramic, we characterized a tetragonal ferroelectric to a tetragonal paraelectric phase transition in which the crystallite anisotropy (or strain) was an important feature to characterize the polar transition. This was also the first report of tetragonal-paraelectric phase in Ca-modifield $\mathrm{PbTiO}_{3}$ ceramics.

[1] B. Jaffe, W.R. Cook, H. Jaffe, Nom-metallic Solids: Piezoelectric Ceramics, 1971.

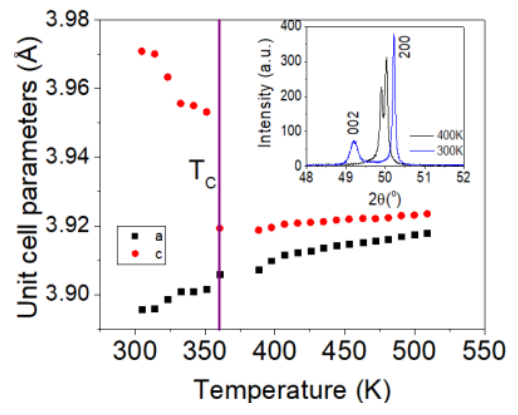

Figure 1. Unit cell parameters for space group $P 4 \mathrm{~mm}$ as a function of temperature. The purple line indicates the paraelectric to ferroelectric phase transition temperature. Inset the 002 and 200 XRD peaks at tetragonal paraelectric $(400 \mathrm{~K})$ and ferroelectric phases $(300 \mathrm{~K})$.

[2] J. Zhao, et al., A combinatory ferroelectric compound bridging simple ABO3 and A-site-ordered quadruple perovskite, Nat. Commun. 12 (2021) 1-9.

[3] F. Regina Estrada, M. Henrique Lente, D. Garcia, The normal to diffuse phase transition crossover from thermal expansion analysis in calcium modified lead titanate, Ferroelectrics. 534 (2018) 50-55.

[4] M. Yashima, R. Ali, Structural phase transition and octahedral tilting in the calcium titanate, Solid State Ionics. 180 (2009) $120-126$.

Keywords: ferroelectricity; piezoelectric ceramic; synchrotron crystallography; perovskite; dielectric materials

The authors wish to thank the Brazilian funding agencies CAPES and CNPq for the financial support. This research used resources of the Brazilian Synchrotron Light Laboratory (LNLS), an open national facility operated by the Brazilian Centre for Research in Energy and Materials (CNPEM) for the Brazilian Ministry for Science, Technology and Innovations (MCTI). We acknowledge the use of XPD and XRD1 beamlines.

Acta Cryst. (2021), A77, C1235 